\title{
What is grouping during binocular rivalry?
}

\author{
Sjoerd M. Stuit *, Chris L. E. Paffen, Maarten J. van der Smagt and Frans A. J. Verstraten
}

Division of Experimental Psychology, Helmholtz Institute, Neuroscience and Cognition Utrecht, Universiteit Utrecht, Utrecht, Netherlands

\section{Edited by:}

Georgios A. Keliris, Max Planck Institute for Biological Cybernetics, Germany

\section{Reviewed by:}

Andreas Bartels, Max Planck Institute for Biological Cybernetics, Germany Ilona Kovacs, Budapest University for Technology and Economics, Hungary

\section{*Correspondence:}

Sjoerd M. Stuit, Division of Experimental Psychology, Helmholtz Institute, Neuroscience and Cognition Utrecht, Universiteit Utrecht,

Heidelberglaan 2, NL-3584 CS

Utrecht, Netherlands.

e-mail:s.m.stuit@uu.nl
During binocular rivalry, perception alternates between dissimilar images presented dichoptically. Although perception during rivalry is believed to originate from competition at a local level, different rivalry zones are not independent: rival targets that are spaced apart but have similar features tend to be dominant at the same time. We investigated grouping of spatially separated rival targets presented to the same or to different eyes and presented in the same or in different hemifields. We found eye-of-origin to be the strongest cue for grouping during binocular rivalry. Grouping was additionally affected by orientation: identical orientations were grouped longer than dissimilar orientations, even when presented to different eyes. Our results suggest that eye-based and orientation-based grouping is independent and additive in nature. Grouping effects were further modulated by the distribution of the targets across the visual field. That is, grouping within the same hemifield can be stronger or weaker than between hemifields, depending on the eye-of-origin of the grouped targets. We also quantified the contribution of the previous cues to grouping of two images during binocular rivalry. These quantifications can be successfully used to predict the dominance durations of different studies. Incorporating the relative contribution of different cues to grouping, and the dependency on hemifield, into future models of binocular rivalry will prove useful in our understanding of the functional and anatomical basis of the phenomenon of binocular rivalry.

Keywords: binocular rivalry, grouping, joint predominance, interocular grouping

\section{INTRODUCTION}

During binocular rivalry, dissimilar images presented dichoptically compete for awareness. As a result, perception varies over time (e.g., Wheatstone, 1838). When large images are engaged in rivalry, perception often consists of a patchwork combination of the competing images. That is, different locations have different perceptual outcomes (e.g., Meenes, 1930), implying that the dominant percept contains parts of both the left and the right eye's image. This patchwork or piecemeal rivalry does not occur when the images are rather small (estimated at $5-7$ min of visual angle in the fovea; Blake et al., 1992). These observations reveal an important characteristic of rivalry, namely that it is a local phenomenon. Investigations into this local nature of rivalry revealed that the size of local rivalry zones scales with eccentricity and may correspond to the size of the receptive fields in the hypercolumns of early visual cortex (Blake et al., 1992).

Although perception during rivalry seems to be determined at a local level, different rivalry zones are not necessarily independent: similar (parts of) images tend to be dominant in perception together. That is, adjacent rivalry zones tend to produce the same dominant percept when neighboring zones share similar features like motion or color, even when this information is distributed across the two eyes (e.g., Whittle et al., 1968; Kovács et al., 1997; Alais and Blake, 1998). For instance, Kovács and her colleagues created rival targets consisting of patchwork combinations of two complex images. Each eye received only part of the originals when they were presented dichoptically. The perceptual outcome during rivalry often consisted of a coherent reconstruction of the original images (Kovács et al., 1997; also see Diaz-Caneja, 1928, translated by Alais et al., 2000). Interestingly, this reconstruction required simultaneous dominance of rivalry zones across both eyes; an effect known as interocular grouping. Similar effects have been found for grouping of spatially separated items (e.g., Whittle et al., 1968). Alais and Blake (1999) demonstrated that similar rival targets that were separated spatially also tend to be dominant at the same time, an effect referred to as joint predominance. They showed that Gestalt grouping cues were effective in increasing the joint predominance of rival targets: joint predominance was larger for parallel and collinear grating-pairs compared to that of orthogonal gratings. Also, correlated contrast modulations of the gratings increased joint predominance in comparison to uncorrelated contrast modulations. These results show how the dominant percept originating from a local rivalry zone is affected by the dominant percept of neighboring rivalry zones. Furthermore, since the effect of joint predominance decreased with angular separation between the rivaling targets, Alais and Blake argued that interactions between lateral connections of the cortical hypercolumns were responsible for their effect.

Together, the above results suggest that the perceptual outcome of two rivaling images is primarily determined at a local level, but that grouping ${ }^{1}$ cues (such as good continuation) affect the local competition: when two adjacent regions contain similar imagecontent, the images tend to be dominant in perception at the same

\footnotetext{
${ }^{1}$ In this study, grouping refers to the simultaneous dominance of two rival targets. When referring to grouping effects found in other studies, we will use the terminology of the original authors (i.e., interocular grouping or joint predominance).
} 
time, even when the image-content is distributed across the two eyes.

The current study has two aims. First, we aim to assess the strength of grouping when rival images are presented to the same versus different eyes and presented in the same versus different hemifields. This allows us to link grouping strength to known aspects of functional visual pathways. As Alais and Blake (1999) suggested, grouping during binocular rivalry might be related to connections at the level of the primary visual cortex. Estimating the grouping strength between targets that have very different cortical representation loci (i.e., represented in different ocular dominance columns and different hemispheres) will provide more insight in the effective connectivity that drives grouping during rivalry.

Our second aim is to elucidate the relative contributions of stimulus-based versus eye-based rivalry during simultaneous dominance of spatially separated targets. Interocular grouping and stimulus-based rivalry both emphasize competition based on image-content over competition based on the eye-of-origin of the images. Theories suggesting that rivalry competition is resolved at "later stages" of visual processing rely on examples of stimulusbased rivalry, such as Flicker-and-Swap-rivalry (Logothetis et al., 1996). These "later stages" are meant as relatively later to those put forward in the many studies emphasizing the low-level nature of binocular rivalry. For example, Blake (1989) argued that monocular neurons are crucial for the initiation of binocular rivalry. The necessity of monocular neurons thus limits rivalry competition to be initiated early in the visual processing hierarchy. Both early and late theories have gained support from psychophysical as well as imaging studies (Polonsky et al., 2000; Tong and Engel, 2001; Silver and Logothetis, 2007). In recent years, these different views have started to converge to the idea that rivalry is resolved at multiple stages along the visual hierarchy (Blake and Logothetis, 2002; Nguyen et al., 2003; Wilson, 2003; Lee, 2004; Freeman, 2005; Silver and Logothetis, 2007). In accordance with this idea, stimulusbased rivalry has been suggested to have a synergetic effect on eye-based dominance periods (Kovács et al., 1997; Lee and Blake, 2004). Determining perceptual dominance durations for different percepts of separate rival targets, presented either to the same or to different eyes, allows us to investigate this in more detail.

Since we know that collinear and parallel gratings tend to group during rivalry, we presented such targets under various spatial arrangements. In our experiments, we presented identical, spatially separated, rival targets (1) to the same or different eyes, and (2) in the same or different hemifields. Estimating the relative strength of grouping two images under these different arrangements allowed us to dissociate low-level, eye-based contributions to perceptual grouping from high-level, pattern-based contributions. Next, we implemented their relative contributions in a simple descriptive model based on the known functional anatomy of primary visual cortex.

\section{MATERIALS AND METHODS OBSERVERS}

A total of 12 observers, including one of the authors (Sjoerd M. Stuit) participated in the study. Eight observers participated in the main experiment and seven, including four observers from the main experiment, participated in a separate version of the experiment (see below). All had normal or corrected to normal vision and all but Sjoerd M. Stuit were naiive as to the purpose of the study. All observers were experienced psychophysical observers and passed a test for stereo vision (TNO test for stereoscopic vision). All observers gave informed consent before participating.

\section{APPARATUS}

Stimuli were created on an Apple Mac Pro computer running system OS-X and Matlab 7.4 with the Psychophysics Toolbox extensions (Brainard, 1997; Pelli, 1997). The stimuli were presented on a linearized LaCie III $22^{\prime \prime}$ at $75 \mathrm{~Hz}$. Observers viewed the stimuli through a mirror stereoscope. The length of the optical path was $57 \mathrm{~cm}$.

\section{STIMULI}

The rival stimuli were two pairs of half-images each consisting of two sine-wave gratings. To initiate rivalry, each interocular pair had orthogonal orientations (Figure 1). The gratings were presented at maximum contrast (98\% Michelson Contrast, spaceaverage luminance: $24.83 \mathrm{~cd} / \mathrm{m}^{2}$ ). The rivaling gratings (spatial frequency $4.1 \mathrm{cpd}$, diameter $1.65^{\circ}$ ) appeared in circular apertures of which the edges were softened by a cosine ramp of $0.2^{\circ}$ of visual angle, and were presented on a random pixel noise background of $98 \%$ (Michelson) contrast (mean luminance $24.83 \mathrm{~cd} / \mathrm{m}^{2}$ ) that was identical in both eyes. The half-images were presented within a white square. We used four basic grating arrangements in our experiment (Figure 1): same orientations in the same hemifield for the same eye: (Figure 1A), same orientations in different hemifields - for the same eye: (Figure 1B), same orientations in the same hemifield - for different eyes: (Figure 1C), and same orientations in different hemifields - for different eyes: (Figure 1D). All presentation conditions were counterbalanced for eye and hemifield. This resulted in each orientation being presented to each eye and in each hemifield equally often. The distance from the fixation point to the center of the target was identical for all targets in all conditions $\left(2.1^{\circ}\right.$ of visual angle). Two versions of the grating-pairs were used. In the main experiment we used horizontal and vertical grating-pairs. Such gratings have been shown to result in perceptual grouping by Alais and Blake (1999). In a second version of the experiment we used oblique gratings, two of which were tilted $45^{\circ}$ clockwise and two that were tilted $45^{\circ}$ counterclockwise from vertical. Comparing the results of two versions of the experiment can provide insight into whether having identical image-content is sufficient for grouping during rivalry.

\section{PROCEDURE}

Observers performed the experiment in a darkened room with their heads stabilized by a chin rest. Before the onset of each trial, observers were presented with two identical pixel noise half-images surrounded by white frames. At the center of each half-image was a fixation point. When ready, an observer initiated a trial by pressing the space bar. Next, two pairs of orthogonal gratings were presented in one of four possible spatial arrangements. Observers performed a $2 \mathrm{AFC}$ perceptual tracking task where they indicated via a key press, whether they perceived two identically oriented gratings (right arrow key), or two orthogonal gratings (left arrow 


\section{A Same eye / Same hemifield}

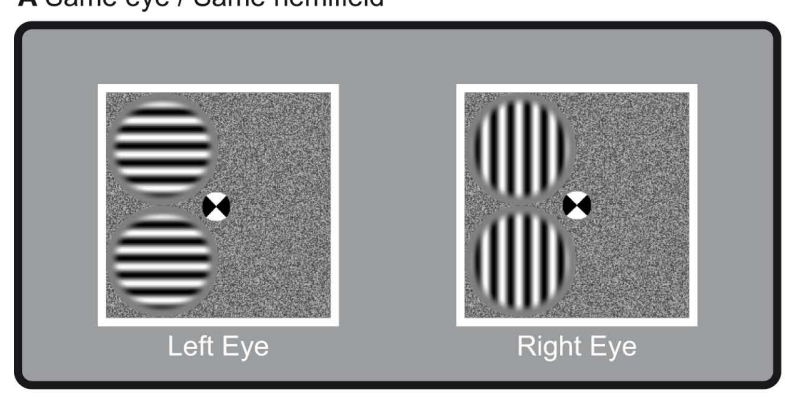

C Different eye / Same hemifield

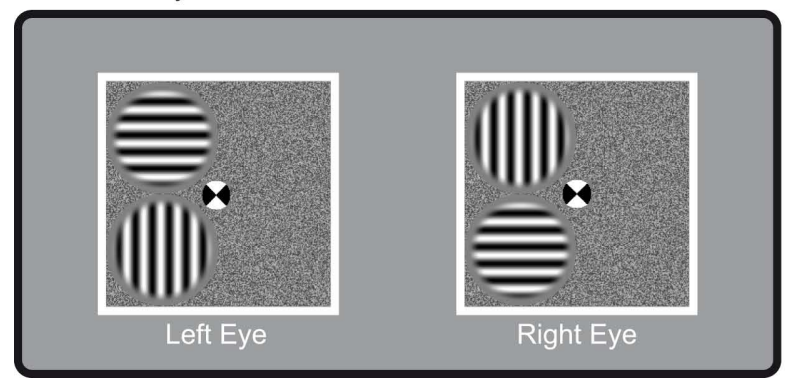

FIGURE 1 | Presentation conditions. The four different stimulus arrangements used. The rival targets were presented such that identical targets were presented (A) in the same hemifield for the same eye; (B) in different hemifields for the same eye; (C) in the same hemifield but for

\section{B Same eye / Different hemifield}

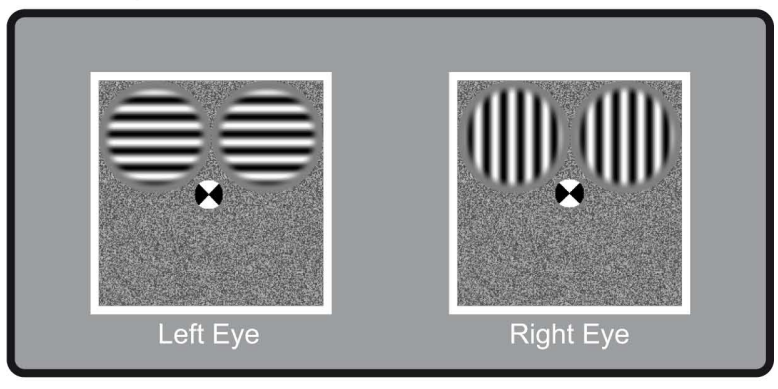

D Different eye / Different hemifield

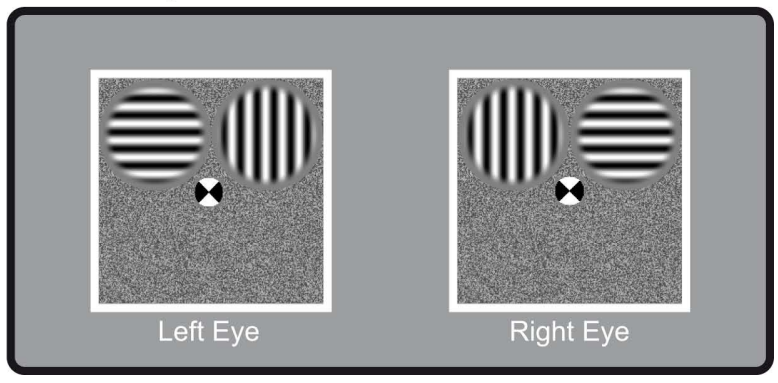

different eyes; or (D) in different hemifields and for different eyes. All presentation conditions were counterbalanced for eye and hemifield. Note that all rival targets had the same distance to each other as well as to the fixation point. key). In case of a mixed percept, observers were still required to make a forced-choice. Note that the use of small gratings kept the occurrence of mixed percepts at a minimum (Blake et al., 1992). Each trial lasted $30 \mathrm{~s}$. After each trial, the rivaling targets were removed from the screen. Observers were instructed to fixate on the fixation point throughout the experiment.

\section{RESULTS}

For our analyses we used two measures for grouping during rivalry: (1) Fractions of simultaneous dominance (i.e., the fraction of time images had the same or different orientations) and (2) epoch durations (i.e., the time an observer had one of these percepts). To get a first impression of the biases for grouping during rivalry we first discuss the fractions for simultaneous dominance of targets with identical cardinal orientations, followed by the underlying dominance epochs that resulted in these fractions for dominance. Subsequently, we will address the data for grouping oblique orientations. Where applicable, the $p$-values were corrected for multiple comparisons.

Our first analyses concerned the fractions of simultaneous dominance for cardinal orientations presented in the same or different hemifields and to the same or different eyes. The fractions were calculated using the time observers actually responded. This means that the duration of each trial that observers did not respond was subtracted from the 30-s trial-duration before calculating the fractions. The fractions of simultaneous dominance were interpreted as an indication of bias toward or away from grouping identical orientations. A fraction of 0.5 means that identical orientations were as often perceived as dissimilar orientations and perception was thus unbiased with respect to grouping.

We compared the fraction of simultaneous dominance of identical orientations across our four presentation conditions; identical orientations presented to (1) the same eye and within the same hemifield, to (2) the same eye but within different hemifields, to (3) different eyes but within the same hemifield, or to (4) different eyes and in different hemifields (Figure 2). A two (eye) by two (hemifield) repeated measures ANOVA revealed a main effect of eye $\left[F(1,7)=45.45, p<0.001, \eta_{\mathrm{p}}^{2}=0.867\right]$, but not of hemifield $\left[F(1,7)=0.66, p=0.689, \eta_{p}^{2}=0.086\right]$. However, an interaction between the two was apparent as well $[F(1,7)=11.19$, $\left.p=0.024, \eta_{\mathrm{p}}^{2}=0.612\right]$. To test the nature of the interaction we compared the effect of hemifield in the same-eye conditions to the different-eye conditions. Hemifield-effects were defined as the difference between the fraction of simultaneous dominance of identical orientations when presented in the same versus different hemifield(s) (i.e., the difference between the white and the dark gray bars in the Same Orientation panels of Figure 2). The result showed that the hemifield-effect differed depending on the (sameand different-) eye condition [paired sample $t$-test: $t(7)=3.34$, $p=0.0245, d=1.18]$. The interaction between eye and hemifield reflects the reversal of this hemifield-effect: when identical orientations were presented to the same eye, presenting those orientations in the same hemifield increased grouping compared to the presentation in different hemifields. However, when identical orientations were split between the eyes, presentation in the same hemifield decreased the fraction of simultaneous dominance. 


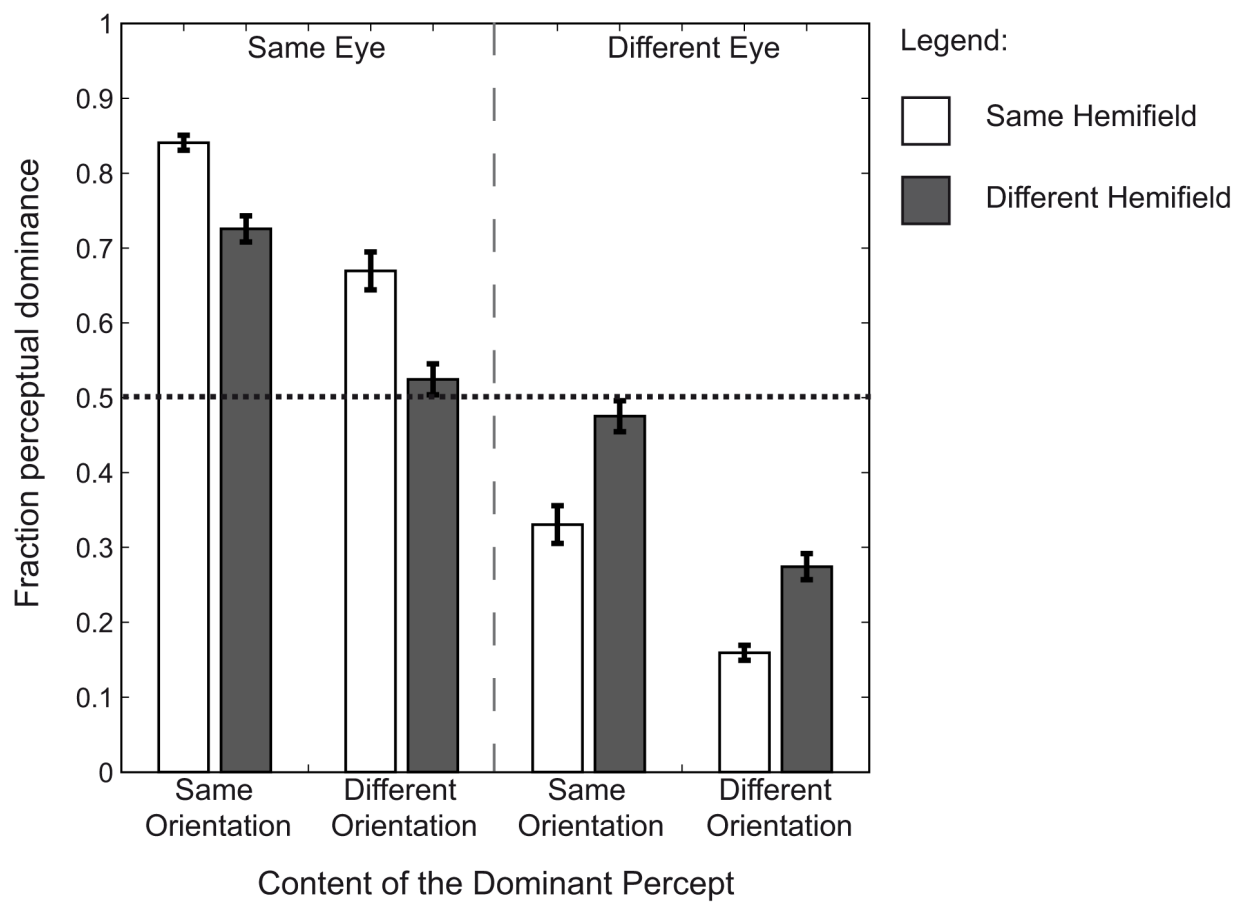

FIGURE 2 | Group means of the dominance fractions. The average fraction of simultaneous dominance across observers for all possible grouped percepts. Error bars represent SEM. Fractions are plotted for each condition. Each bar represents the fraction of dominance for two targets. The dotted line represents unbiased dominance. The results show that when identical rival targets are presented to the same eye, there is a bias toward orientation-based grouping. This is not the case when identical targets are split between the eyes. The bias toward grouping is further increased when identical targets are presented within the same hemifield as well as to the same eye. However, when identical targets are presented to different eyes, the bias is decreased (a bias away from grouping) for presentation in the same compared to different hemifields. These results suggest a strong preference for visual information presented to one eye to be simultaneously dominant.
Each fraction of simultaneous dominance of identical orientations was subsequently tested for a bias toward orientationbased grouping using paired samples $t$-tests. The results show that when identical orientations were presented to the same eye, there was a bias toward grouping for both the same and different hemifield conditions $[t(7)=16.83, p<0.001, d=5.95$; $t(7)=6.47, p=0.001, d=2.29$ respectively]. However, when the identical orientations were presented to different eyes, there was no bias toward grouping based on orientation [same hemifield, biased away from orientation-based grouping: $t(7)=-3.35, p=0.048, d=-1.18$; different hemifields, unbiased: $t(7)=-0.60, p=0.965, d=-0.21]$. These results show that there is only a bias toward grouping identical orientations when they are presented to the same eye.

The analysis of the fractions of simultaneous dominance suggests that grouping during rivalry primarily occurs between targets presented to the same eye. However, identical fractions can result from very different distributions of dominance epochs. To get a more detailed picture of the effect of grouping on perceptual dominance, we compared the dominance durations for each combination of grouped targets (identical or different orientations).

To calculate the dominance durations we used the median duration (per condition per observer) to correct for the known skewed distribution of dominance epochs (Levelt, 1967). In addition, large individual differences in dominance durations are known to be common as well (e.g., Aafjes et al., 1966). To correct for the latter, all durations were normalized to each observers' average median dominance duration across all trials, percepts (simultaneous dominance of the same of different oriented gratings) and conditions.

Throughout the experiment, simultaneous dominance of two targets can reflect grouping based on multiple cues: eye-of-origin, hemifield, and orientation (Figure 3). The comparison of the average duration of each of these perceptual outcomes can be used to estimate the strength of each grouping cue. Epoch durations for each perceptual outcome were compared using a two (eye) by two (orientation) by two (hemifield) repeated measures ANOVA. We found a main effect for eye $[F(1,7)=61.54$, $\left.p<0.001, \eta_{\mathrm{p}}^{2}=0.898\right]$ as well as for orientation $[F(1,7)=46.14$, $\left.p<0.001, \eta_{\mathrm{p}}^{2}=0.868\right]$, but not for hemifield $[F(1,7)=1.46$, $\left.p=0.461, \eta_{\mathrm{p}}^{2}=0.172\right]$. As was true for the analysis of the fraction simultaneous dominance of identical targets, we found an interaction between eye and hemifield $[F(1,7)=11.35, p=0.024$, $\left.\eta_{\mathrm{p}}^{2}=0.619\right]$. This interaction reflects the difference in the hemifield-effect when the same orientations were presented to the same eye versus when they were presented to different eyes [paired sample $t$-test: $t(7)=3.37, p=0.024, d=1.19]$. No interaction between orientation and hemifield $\left[F(1,7)=0.07, p=0.960, \eta_{\mathrm{p}}^{2}=\right.$ $0.010]$, orientation and eye-of-origin $[F(1,7)=2.30, p=0.316$, 


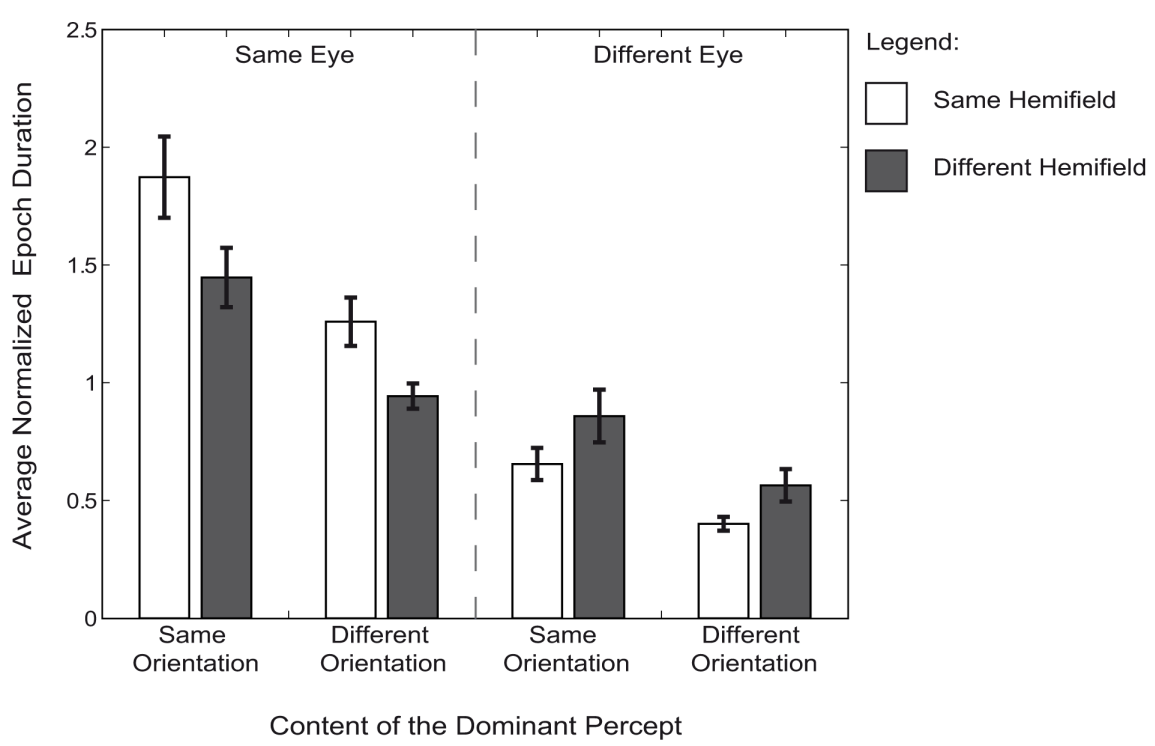

FIGURE 3 | Average epoch duration. Average normalized median durations of each particular perceptual outcome are represented for each condition. All durations are normalized to each observer's median epoch duration across all trials, percepts, and conditions. Error bars are SEM. Each bar represents the dominance duration that two targets were dominant at the same time. The durations of grouping two targets are separated by the targets' eye-of-origin, the hemifield in which they were presented and their orientation. $\left.\eta_{\mathrm{p}}^{2}=0.248\right]$ or three-way interaction was found $[F(1,7)=0.76$, $\left.p=0.567, \eta_{\mathrm{p}}^{2}=0.097\right]$.

Recent evidence suggests eye-based and image-based influences on binocular rivalry vary over time (Bartels and Logothetis, 2010). Their results suggest epoch duration may become shorter as rivalry continues. Moreover, the first second of rivalry competition has been argued to be fundamentally different from the remaining rivalry period (Carter and Cavanagh, 2007). Using relatively short epoch durations, the first few seconds of each rivalry period may be overrepresented in our data. However, we found no effect of time on the different contributions to grouping in our paradigm statistically nor did we find any apparent trend to the influence of time on these contributions. The only hint to in temporal effects was a slight increase in dominance epoch durations near the end of the rivalry periods compared to the beginning of the trials.

The analysis of the fractions of simultaneous dominance suggested grouping is primarily eye-based; there was no bias toward grouping identical orientations presented to different eyes. However, the analysis of the epoch durations did show an effect of orientation on grouping: identical orientations are grouped for longer durations than dissimilar orientations. Importantly, this effect was not dependent on the eye-of-origin of the grouped targets (e.g., no interaction). This suggests a grouping effect of orientation irrespective of whether the images are presented to the same eye or not. The differential effects of grouping based on eye-of-origin and orientation cannot be fully disentangled by comparisons of the fractions of dominance. However, the analyses of epoch durations appear to be a much more sensitive measure to investigate the different grouping cues. This difference between the fraction and the epoch results is likely to stem from a difference in the underlying distributions of dominance durations; distributions for within-eye grouping were much broader than for between-eye grouping. These characteristics of the underlying distributions are lost in the comparison of the fractions of grouping during dominance. The median durations, however, are less affected by these differences in the underlying distributions.

The next step in our analysis is to quantify the respective contributions of the eye-of-origin and orientation cues for grouping (Figure 4A). The hemifield condition was ignored since the repeated measures ANOVA showed no main effect for hemifield on grouping targets during rivalry. Therefore, simultaneous dominance of two targets can be based on a single cue for grouping (a shared eye-of-origin or a shared orientation), on two grouping cues (a shared eye-of-origin and a shared orientation), or no grouping cues at all (simultaneous dominance of targets with different orientations and presented to different eyes). We tested whether the number of grouping cues affected the average median duration of a particular percept. Using paired sample $t$-tests, we found that the duration of simultaneous dominance based on a single grouping cue is longer than when there are no grouping cues at all [orientation cue: $t(7)=4.21$, $p=0.020, d=1.49$; eye-of-origin cue: $t(7)=10.49, p<0.001$, $d=3.71$ ]. Also, eye-of-origin provides a stronger grouping cue than orientation $[t(7)=4.39, p=0.016, d=1.55]$. Finally, when both cues are present, the duration of simultaneous dominance is longer than for any single cue alone [compared to orientation: $t(7)=4.98, p=0.008, d=1.76$; compared to eye-of-origin: $t(7)=3.89, p=0.030, d=1.37]$. These results show the effectiveness of both grouping cues on the duration of particular percept as well as the relative strength of each cue.

Our final analysis is concerned with the observation that synergistic interactions among neighboring rivalry zones reinforce perception of coherent patterns during rivalry (Kovács et al., 1997; Blake, 2001; Lee and Blake, 2004). Our results for grouping 


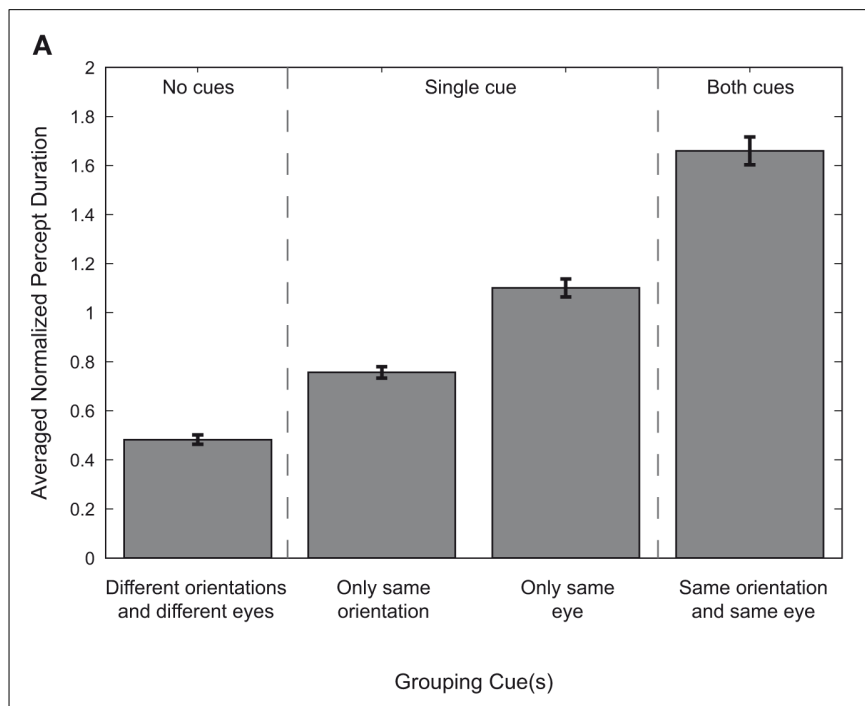

FIGURE 4 | Percept durations per grouping cue. The average median duration of each particular perceptual outcome, represented for each grouping cue. All durations are normalized to each observer's average median epoch duration of all trials, percepts, and conditions. Error bars represent SEM. The data are arranged based on the number of grouping

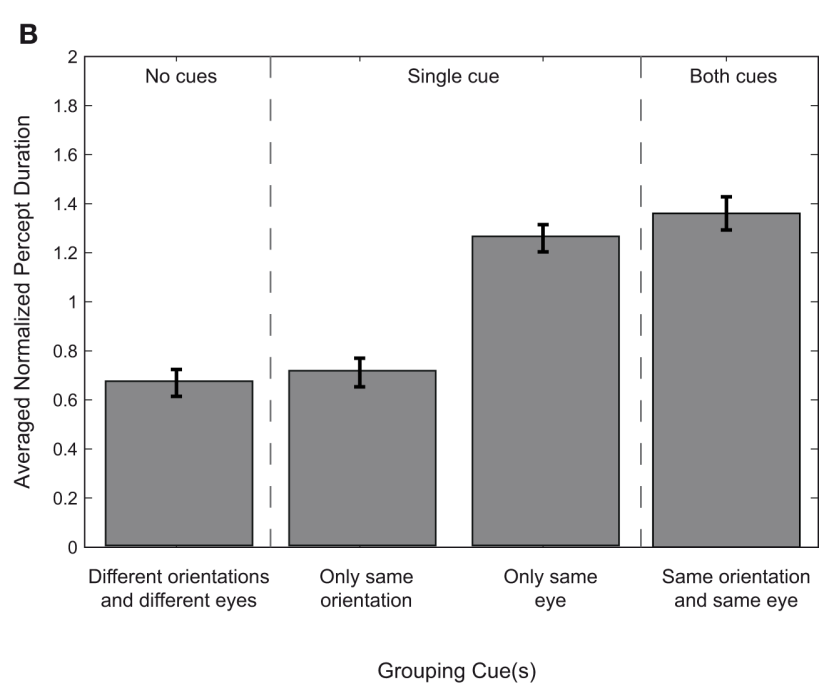

cues associated with each perceptual outcome. The results are plotted as a hierarchy to illustrate the relative potency of certain grouping cues over others. (A) Displays the results for our main experiment using cardinal orientations. (B) Displays the results for grouping when oblique orientations are used. cardinal orientations confirm this by showing that different cues can combine to have a synergistic effect on the duration of grouping during rivalry. To test the nature of this synergistic effect, we first took the duration of simultaneous dominance of a horizontal and a vertical oriented target presented to different eyes (the left most bar in Figure 4A) as the baseline duration for grouping. Next, we subtracted this baseline from the durations of simultaneous dominance based on either one or more grouping cues. These difference-scores show the additional contribution to the duration of grouping associated with each cue (Figure 5A). A paired sample $t$-test showed that the sum of the added durations of simultaneous dominance based on image-content and eye-of-origin is not significantly different from the duration of simultaneous dominance when both these cues are present $[t(7)=1.52, p=0.173, d=0.54]$. This finding concurs with the lack of an interaction between the eye-of-origin and the orientation of the rival targets (see above). In sum, this suggests that the cue effects are independent, and act additively on dominance durations.

The effectiveness of the orientation cue for grouping described above is applicable to cardinal orientations. The same analysis on the data for the experiment using oblique orientations also shows an eye-of-origin effect on grouping rivaling targets $[t(6)=3.09$, $p=0.04, d=1.17$; Figures $4 \mathbf{B}$ and $\mathbf{5 B}]$. Note that the effect is similar in magnitude compared to the experiment using cardinal orientations. However, for oblique orientations, we found no effect of the orientation cue to grouping $[t(6)=0.71, p=0.757, d=0.27]$. In addition, analyses of the fractions of dominance when the rivaling grating-pairs were oblique (as is represented for cardinal orientations in Figure 2) only showed a significant effect of eye-oforigin $\left[F(1,6)=13.69, p<0.01, \eta_{\mathrm{p}}^{2}=0.695\right]$ and the interaction between eye-of-origin and hemifield $[F(1,6)=10.58, p=0.017$, $\left.\eta_{\mathrm{p}}^{2}=0.638\right]$, but no effect of orientation $[F(1,6)=1.46, p=0.272$, $\left.\eta_{\mathrm{p}}^{2}=0.196\right]$. These results show that cardinal orientations are more readily grouped than oblique orientations during rivalry dominance. Furthermore, the lack of grouping identical oblique orientations suggests that having identical image-content is not sufficient for grouping during rivalry dominance.

\section{GENERAL DISCUSSION}

We investigated perceptual grouping of two spatially separated rival targets under a variety of spatial arrangements. Identical rival targets were presented to the same or to different eyes, and within the same or in different hemifields. For cardinal orientations, we found a bias toward grouping when identical orientations were presented to the same eye, but no such bias was evident when identical orientations were presented to different eyes. Moreover, for oblique orientations, grouping during dominance was only affected by eye-of-origin. These results show that eye-of-origin is an important factor for grouping similar orientations during binocular rivalry. The distribution of the targets across the visual field also affected grouping with respect to eye-of-origin. Grouping occurred more often for images presented to the same eye when the images were in the same hemifield. The opposite was true for images that were split between the eyes. That is, images in the same hemifield were grouped less often. The modulation of grouping effects by the distribution of the targets across the visual field appears independent of orientation.

The overall occurrence of simultaneous dominance of two targets during rivalry was not biased toward grouping identical orientations across the eyes. However, for cardinal orientations, the duration of grouping was affected by orientation irrespective of whether the images were presented to the same eye or not. Our results suggest that eye-of-origin and orientation provided independent cues for grouping during rivalry, with eye-of-origin being the superior cue. It has before been argued that synergistic 


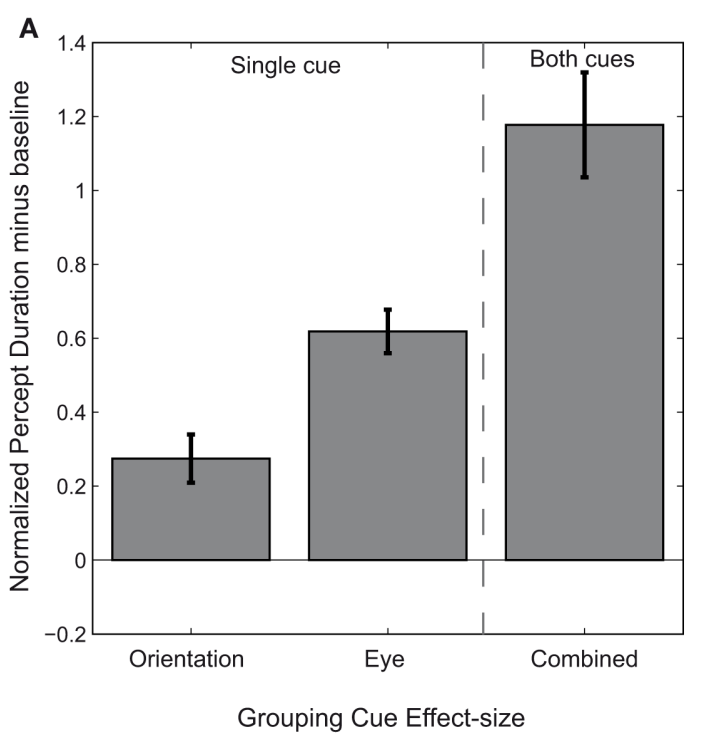

FIGURE 5 | Effect sizes of the different grouping cues. The data from Figure 4, displayed as difference scores by subtracting the baseline dominance duration: the duration of dominance when no grouping cues are present. The difference-scores represent the added percept duration and are represented as a function of cue effect.

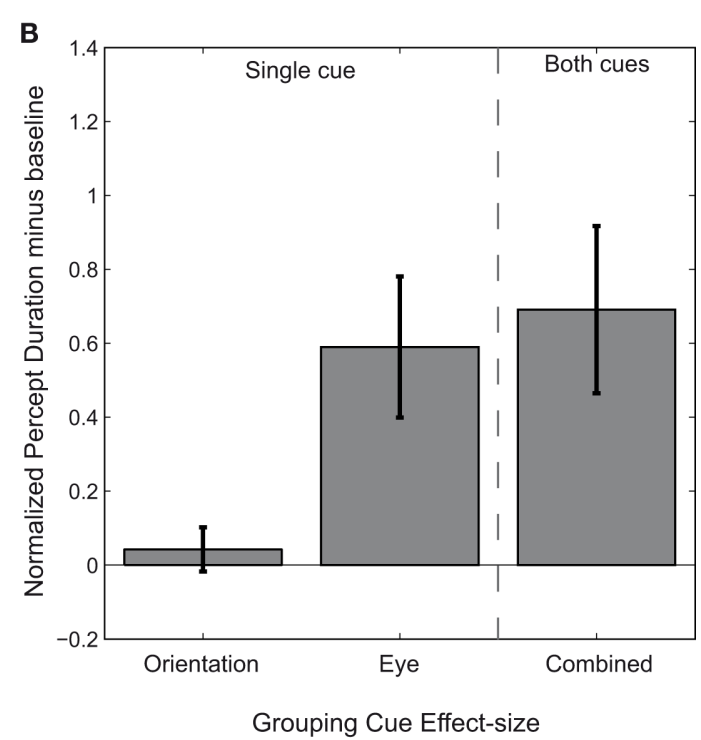

Note that the sum of both single cues does not differ from the dominance duration when both cues are simultaneously present. We suggest the cue effects are independent and have additive effects on dominance durations. (A) Show the data for cardinal orientations. (B) Shows the data for oblique orientations. interactions among neighboring rivalry zones reinforce the perception of coherent patterns during rivalry (Kovács et al., 1997; Blake, 2001; Lee and Blake, 2004). We present quantitative evidence for a synergy between grouping based on image-content, and eye-of-origin. More specifically, our results show that this synergetic effect is additive in nature. However, we only found an effect of image-content for rivaling grating-pairs with cardinal orientations; no such effect was found for oblique orientations. The difference between these results likely stems from the lack of co-linearity when rivalry is between oblique grating-pairs. Both psychophysical and physiological studies have shown that facilitation of a flanking line on a target is largest when the lines are collinear (Kapadia et al., 1995). However, non-collinear parallel orientations have also been shown to group during rivalry dominance (Alais and Blake, 1999). Since the identically oriented oblique gratings are not collinear but nevertheless parallel, one would expect the grouping effects for the oblique gratings to possibly be smaller, but still present. In the present study, however, no such orientation-based grouping was found for oblique orientations. Therefore, our results suggest that the grouping effects for the oblique gratings, if present at all, are very small. We currently do not have an explanation why we found no orientation-based grouping for oblique grating-pairs. One possibility is that parallelism is not a strong cue for grouping when the rival targets are not aligned on an axis orthogonal to their orientation.

The data from this study can be used to calculate the relative contributions for all cues affecting grouping during rivalry: eyeof-origin, orientation, and hemifield. These relative contributions of each are taken directly from the normalized epoch durations as reported in Figure 3: the average normalized median durations of simultaneous dominance of two rival targets. The contributions are implemented as weights in Figure 6: a schematic representation of the cortical hypercolumns in early visual cortex. The cartoon is made for descriptive purposes only and we refrain from making strong statements about the anatomical connections underlying the (grouping) weights. A first thing to notice is that grouping is strongest for items presented to the same eye (Figure 6, connections A-D). Whether the items are identical or not, and whether they are processed in the same hemisphere or not, does not affect the generality of the effect. Also, eye-based grouping of two targets is stronger when they are presented in the same hemifield (Figure 6, connection A versus $C$ and connection $B$ versus $D)$. This fits well with the decrease in joint predominance with increasing lateral separation as reported by Alais and Blake (1999), and with the decrease in connection strength as a function of increasing cortical distance (Das and Gilbert, 1995; Bosking et al., 1997). However, grouping of targets between the eyes is stronger when they are presented in different hemifields (as compared to presentation in the same hemifield; Figure 6, connections $\mathrm{G}$ and $\mathrm{H}$ versus connections $\mathrm{E}$ and $\mathrm{F}$ ). At first sight, this result is counterintuitive, since connections between hemispheres are longer than connections within a hemisphere. We speculate that this result indicates that connections between different eyes are more inhibitory (leading to less grouping) within a hemisphere, compared to between hemispheres. In conclusion, we can draw a hierarchy of different visual structures involved in grouping: grouping of both similar and different orientations is (1) strongest across hypercolumns receiving input from a single eye and hemifield, (2) weaker across hypercolumns from a single eye between hemifields, (3) weaker across hypercolumns from different eyes and hemifields, and (4) weakest across hypercolumns from a single eye between hemifields. 


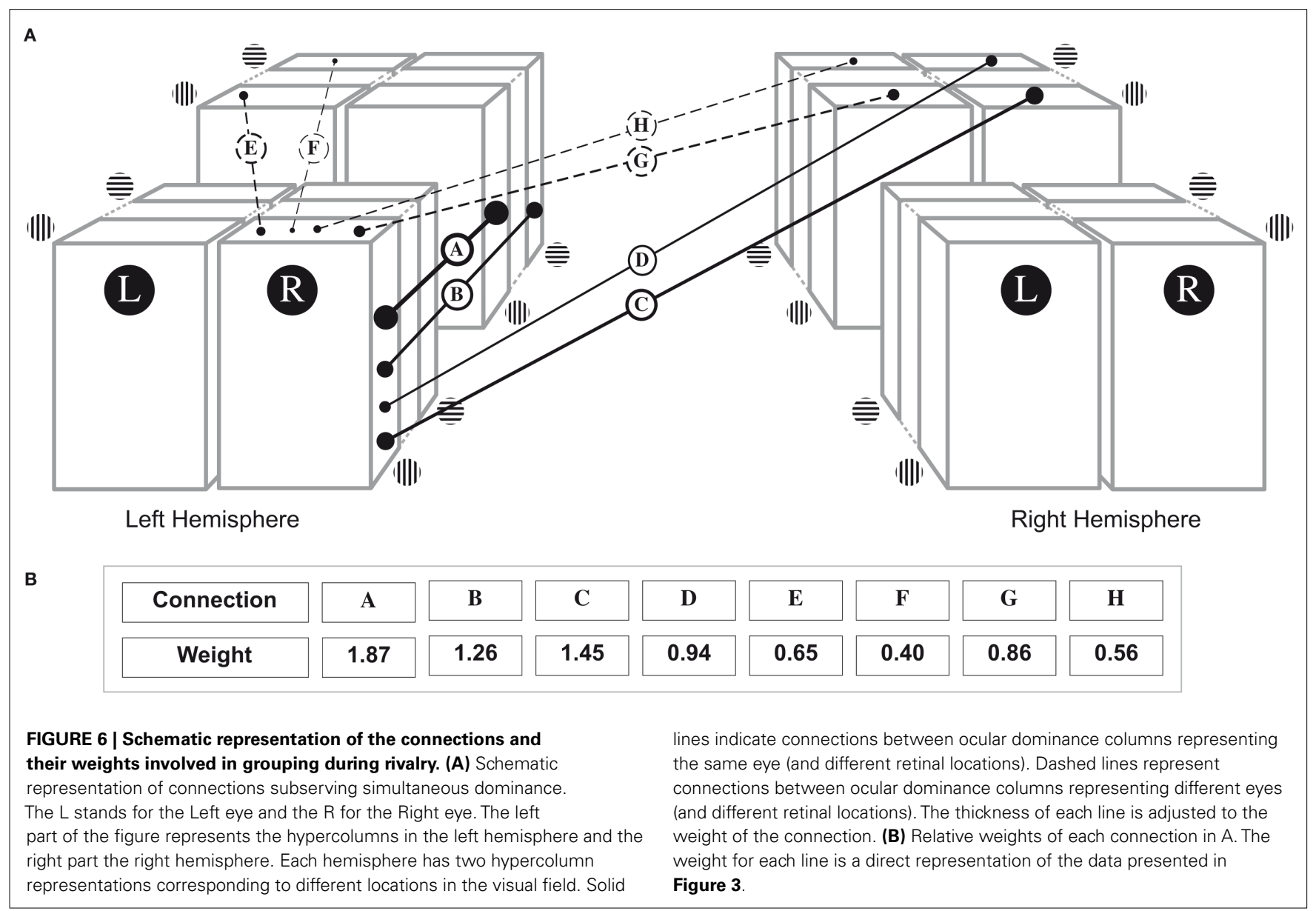

Although the above model is first and foremost descriptive for our results, we can use these weights for grouping to try and make quantitative predictions. For example, in their experiment 1, Alais and Blake (1999) measured joint predominance for pairs of collinear, parallel, and orthogonal gratings that rivaled with noise patches. The grating-pairs were presented to the same eye but in different hemifields. As such, the results of their parallel and collinear conditions are comparable to our results for perceiving identical orientations presented to the same eye and in different hemifields (i.e., connection $\mathrm{C}$ in Figure 6). The results for their orthogonal condition are comparable to our results for perceiving different orientations presented to the same eye and in different hemifields (i.e., connection D in Figure 6). Our results would then suggest the strength of the links between the collinear and the parallel grating-pairs to correspond to the weight of 1.45 and the orthogonal pair to correspond to the weight of 0.94. Based on these weights we would expect the grouping effect for the collinear and parallel gratings to be 1.54 times larger than that for the orthogonal pair. From Alais and Blake's Figure 1B, we see that the fraction of grouping for the orthogonal pair is about 0.31 . The fractions for grouping the parallel and collinear gratingpairs are about 0.53 and 0.42 respectively. The average fraction of these two conditions (0.475) is thus 1.53 times larger than for the orthogonal condition, almost exactly the same ratio as that follows from our results. Interestingly, the images suppressed during simultaneous dominance of the different grating-pairs differed substantially between their study and ours. While we used a second grating pair, Alais and Blake used noise patches to rival with their gratings. Since their results are quantitatively similar to ours, the content of the suppressed images does not appear to affect the relative strength of grouping during dominance. This suggests another interesting feature of grouping during rivalry: the strength of grouping is based on the currently dominant images, not the suppressed ones. Note that the difference between grouping for parallel and for collinear gratings shown by Alais and Blake (1999) suggests that the collinear grating-pairs may have had the greatest influence on our grouping results. Since we did not have observers dissociated between the orientations in the dominant percept we cannot test this directly. However, with collinear being a stronger grouping cue than parallel only, this is very likely the case.

Our results also make predictions about the spread of traveling waves typically seen during rivalry alternations (Wilson et al., 2001). From our results we would expect a difference between waves traveling within one hemifield as compared to the across hemifields. Since our data suggests the linking of neighboring rivalry zones to be stronger within the same hemifield (assuming eye-based dominance of an image) we expect traveling waves to spread faster within hemifields (e.g., vertical spreading) compared to across hemifields (e.g., horizontal spreading). To our knowledge, this has not yet been tested, although the data may already be 
available (e.g., Lee et al., 2005). This is not unexpected since models of binocular rivalry do not typically involve any consideration as to which hemifield or -fields the image(s) is presented in, for instance, Blake (1989), Wilson (2003), Ashwin and Lavric (2010). Our results show that incorporation of the hemifield-effect may be critical in predicting dominance durations during binocular rivalry.

To summarize, we investigated grouping of spatially separated rival targets presented to the same or to different eyes and presented in the same or in different hemifields. We found that:

- Eye-of-origin is the strongest cue for grouping during binocular rivalry.

- Identical cardinal orientations are grouped in dominance for longer periods than dissimilar orientations.

\section{REFERENCES}

Aafjes, M., Hueting, J. E., and Visser, P. (1966). Individual and interindividual differences in binocular retinal rivalry in man. Psychophysiology 3, $18-22$.

Alais, D., and Blake, R. (1998). Interactions between global motion and local binocular rivalry. Vision Res. 38 , 637-644.

Alais, D., and Blake, R. (1999). Grouping visual features during binocular rivalry. Vision Res. 39, 4341-4353.

Alais, D., O'Shea, R. P., Mesana-Alais, C., and Wilson, I. G. (2000). On binocular alternation. Perception 29, 1437-1445.

Ashwin, P., and Lavric, A. (2010). A low-dimensional model of binocular rivalry using winnerless competition. Physica D 239, 529-536.

Bartels, A., and Logothetis, N. K. (2010). Binocular rivalry: a time dependence of eye and stimulus contributions. $J$. Vis. 10, 1-14.

Blake, R. (1989). A neural theory of binocular rivalry. Psychol. Rev. 96, 145-167.

Blake, R. (2001). A primer on binocular rivalry, including current controversies. Brain Mind 2, 5-38.

Blake, R., and Logothetis, N. K. (2002). Visual competition. Nat. Rev. Neurosci. 3, 13-21.

Blake, R., O'Shea, R. P., and Mueller, T. J. (1992). Spatial zones of binocular rivalry in central and peripheral vision. Vis. Neurosci. 8, 469-478.

Bosking, W. H., Zhang, Y., Schofield, B., and Fitzpatrick, D. (1997). Orientation selectivity and the arrangement of horizontal connections in tree shrew striate cortex. J. Neurosci. 17, 2112-2127.

Brainard, D. H. (1997). The Psychophysics toolbox. Spat. Vis. 10, 433-436.

Carter, O., and Cavanagh, P. (2007). Onset rivalry: brief presentation isolates an early independent phase of perceptual competition. PLoS ONE 2, e343. doi:10.1371/journal.pone. 0000343

Das, A., and Gilbert, C. D. (1995). Longrange horizontal connections and their role in cortical reorganization revealed by optical recording of the cat primary visual cortex. Nature 375, 780-784.

Diaz-Caneja, E. (1928). Sur l'alternance binoculaire. Ann. Oculist. 165, 721-731.

Freeman, A. W. (2005). Multistage model for binocular rivalry. $\mathrm{J}$. Neurophysiol. 94, 4412-4420.

Kapadia, M. K., Ito, M., Gilbert, C. D., and Westheimer, G. (1995). Improvement in visual sensitivity by changes in local context: parallel studies in human observers and in V1 of alert monkeys. Neuron 15, 843-856.

Kovács, I., Papathomas, T. V., Yang, M., and Fehér, A. (1997). When the brain changes its mind, Interocular grouping during binocular rivalry. Proc. Natl. Acad. Sci. U.S.A. 93, 15508-15511.

Lee, S. (2004). Binocular battles on multiple fronts. Trends Cogn. Sci. (Regul. Ed.) 8, 148-151.

Lee, S. H., and Blake, R. (2004). A fresh ing binocular rivalry. Vision Res. 44, 983-991. look at interocular grouping dur-

- Identical image-content alone is not sufficient for grouping during dominance.

- Eye-based and orientation-based grouping are independent effects and additive in nature.

- Grouping within and between hemifields will increase or decrease depending on the presence of the eye-of-origin grouping cue.

The different contributions to perceptual grouping can be easily quantified and used to make predictions on dominance durations in other studies. Although beyond the scope of the present paper, incorporating these weights, including the dependency on hemifield, into future models of binocular rivalry, may prove useful in our understanding of the functional and anatomical basis of the phenomenon.

Lee, S. H., Blake, R., and Heeger, D. J. (2005). Traveling waves of activity in primary visual cortex during binocular rivalry. Nat. Neurosci. 8, 22-23.

Levelt, W. J. M. (1967). Note on the distribution of dominance times in binocular rivalry. Br. J. Psychol. 58, 143-145.

Logothetis, N. K., Leopold, D. A., and Sheinberg, D. L. (1996). What is rivaling during binocular rivalry? Nature 380, 621-624.

Meenes, M. (1930). A phenomenological description of retinal rivalry. Am. J. Psychol. 42, 260-269.

Nguyen, V. A., Freeman, A. W., and Alais, D. (2003). Increasing depth of binocular rivalry suppression along two visual pathways. Vision Res. 43, 2003-2008.

Pelli, D. G. (1997). The VideoToolbox software for visual psychophysics: transforming numbers into movies. Spat. Vis. 10, 437-442.

Polonsky, A., Blake, R., Braun, J., and Heeger, D. J. (2000). Neuronal activity in human primary visual cortex correlates with perception during binocular rivalry. Nat. Neurosci. 3, 1153-1159.

Silver, M., and Logothetis, N. K. (2007). Temporal frequency and contrast tagging bias the type of competition in interocular switch rivalry. Vision Res. 47, 532-543.

Tong, F., and Engel, S. A. (2001). Interocular rivalry revealed in the human cortical blindspot representation. Nature 411 , 195-199.

Wheatstone, C. (1838). On some remarkable, and hitherto unobserved, phenomena of binocular vision. Philos. Trans. R. Soc. Lond. B Biol. Sci. 37, 1-394.

Whittle, P., Bloor, D. C., and Pocock, S. (1968). Some experiments on figural effects in binocular rivalry. Percept. Psychophys. 4, 183-188.

Wilson, H., Blake, R., and Lee, S. (2001). Dynamics of travelling waves in visual perception. Nature 412, 907-910.

Wilson, H. R. (2003). Computational evidence for a rivalry hierarchy in vision. Proc. Natl. Acad. Sci. U.S.A. 100, 14499-14503.

Conflict of Interest Statement: The authors declare that the research was conducted in the absence of any commercial or financial relationships that could be construed as a potential conflict of interest.

Received: 19 July 2011; paper pending published: 05 August 2011; accepted: 30 September 2011; published online: 18 October 2011.

Citation: Stuit SM, Paffen CLE, van der Smagt $M J$ and Verstraten FAJ (2011) What is grouping during binocular rivalry? Front. Hum. Neurosci. 5:117. doi: 10.3389/fnhum.2011.00117

Copyright (C) 2011 Stuit, Paffen, van der Smagt and Verstraten. This is an openaccess article subject to a non-exclusive license between the authors and Frontiers Media SA, which permits use, distribution and reproduction in other forums, provided the original authors and source are credited and other Frontiers conditions are complied with. 